\title{
Acute MI Detection Derived From ECG Parameters Distribution
}

\author{
Alfonso Aranda ${ }^{1,2}$, Joël Karel $^{1}$, Pietro Bonizzi ${ }^{1}$, Ralf Peeters ${ }^{1}$ \\ ${ }^{1}$ Department of Data Science and Knowledge Engineering, Maastricht University (Netherlands) \\ ${ }^{2}$ Medtronic Bakken Research Center, Maastricht (Netherlands)
}

\begin{abstract}
Several studies in the past have evaluated the use of different ECG-based features to diagnose acute myocardial infarction (AMI). This was generally done by looking at how well a feature reflects differences between baseline (no AMI) and AMI situations. This approach tends to overlook the progress of AMI and to underestimate false positives when implemented into a continuous monitoring setting and therefore appears inadequate for it. This has hindered the adoption of those methods in the clinical practice. In this research, we present a novel set of parameters for the dynamic assessment of AMI condition. Those parameters are obtained by analyzing the changes over time in the distribution properties of ECG-based features.
\end{abstract}

\section{Introduction}

Acute myocardial infarction (AMI), also called myocardial ischemia, is the initial step of myocardial infarction and results from an imbalance between oxygen supply and demand. Morbidity and mortality from AMI are significantly reduced when symptoms (chest pain, shortness of breath, etc.) are recognized early enough, reducing time to treatment. The ECG is a routine part of the diagnostic work-up of patients with suspected AMI. The more common ECG manifestations of AMI are ST elevation, ST depression and T-wave changes [1].

In the past three decades, there have been plenty of research focused on building computational methods to analyze ECG signals for the prediction and detection of AMI. However, the proposed methods suffer from several limitations and shortcomings that have resulted in low to no adoption of such methods in the clinical practice [2]. The main problem has been the high number of false alarms and the burden that they put on medical personnel. One of the main causes for the high false alarms rate has to do with the use of sensitivity and specificity as performance measures, evaluating how well the method is able to differentiate between baseline (no AMI) and AMI conditions using two single time point measurements. This does not account for the dynamic nature of both the AMI condition and noise. We believe that dynamic assessment (progress) of AMI will allow to tackle the problem of false positives increasing the adoption of automatic methods for the diagnosis of AMI in the clinical practice.

Recently, we used vectorcardiography (VCG) [3][4], to diagnose myocardial infarction [5] and AMI [6]. In [6], we used a reduced set of ECG leads to diagnose AMI, which would be more convenient in situations of longterm, ambulatory, and home monitoring. In this research, we used the same set of reduced ECG leads and the same set of features and analyzed the changes over time in their distribution properties.

\section{Materials and Methods}

\subsection{Database}

We used the PhysioNet STAFF III database [7][8] as data source. This database consists of standard 12-lead ECG recordings from 104 patients with stenotic coronary arteries, who received elective percutaneous transluminal coronary angiography (PTCA) in one of the major coronary arteries, thus simulating AMI episodes in patients. From the 104 patients' recordings, 6 patients were excluded because of either problems in the 12-lead configuration or noise. The database contains 5 minutes baseline ECG recordings (pre-inflation) acquired at rest and in supine position prior any catheter insertion. Also, it contains inflation ECG recordings (AMI simulated event) with a duration up to 5 minutes, being the mean inflation time 4 minutes and 23 seconds. We considered those recordings as AMI situation in our analysis.

\subsection{Reduced Lead System}

To evaluate the presence of AMI, we used the same reduced lead system as in [6]. This lead system is a 3-lead ECG system composed of the subset of leads V1, V4 and III from the standard 12-lead ECG. Those leads were found to be optimal for the diagnosis of AMI in [6] and therefore we used them as well in the present work.

\subsection{Distribution Parameters}

In [6], we selected a subset of 7 out of 328 features (ST elevation and vectorcardiography features) to diagnose AMI condition. Table 1 shows the description of 
those features. For a detailed definition of these features and their computation, refer to [6]. In this work, we analyzed the changes over time in the distribution properties of those features. For that, we calculated the standard deviation, kurtosis, skewness and symmetry during both baseline and AMI conditions. Standard deviation, kurtosis and skewness were calculated with the standard formulation found in the literature, while symmetry was calculated as the difference between the mean and the median of the feature distribution $X$ (equation 1).

$$
\text { Symmetry }[X]=\operatorname{mean}[X]-\operatorname{median}[X]
$$

Table 1. Set of ECG-based features used.

\begin{tabular}{|c|c|c|}
\hline Feature & Type & Description \\
\hline T_PA_SP & VCG & $\begin{array}{l}\text { T-wave loop perimeter/area ratio in } \\
\text { the sagittal plane. }\end{array}$ \\
\hline T_AREA_SP & VCG & T-wave loop area in the sagittal plane. \\
\hline T_STD_Z & $\mathrm{VCG}$ & $\begin{array}{l}\text { T-wave standard deviation in z VCG } \\
\text { component. }\end{array}$ \\
\hline QRS_PA_3D & VCG & QRS loop 3D perimeter/area ratio. \\
\hline QRS_AVG_X & VCG & $\begin{array}{l}\text { QRS average vector length of VCG x } \\
\text { component. }\end{array}$ \\
\hline STE_III & STE & ST elevation in lead III. \\
\hline STE_V4 & STE & ST elevation in lead V4. \\
\hline
\end{tabular}

\subsection{Signal Processing}

In order to have all the features within the same value range and before doing any calculation, we normalized them using the $\mathrm{z}$ - transform (equation 2 ).

$$
x_{\text {norm }}=\frac{x-\bar{x}}{\sigma}
$$

where $x$ is the input signal, $\bar{x}$ is the signal mean and $\sigma$ is the signal's standard deviation.

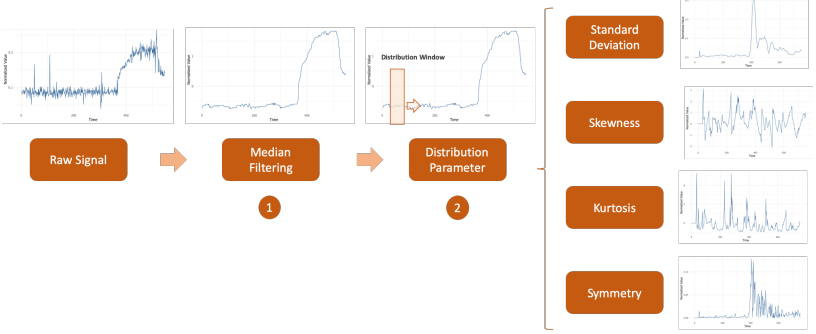

Figure 1 - Two-step approach process to calculate distribution parameter values from one of the features (STE_III) and for one of the patients in the dataset.

Once we had the features normalized, we did a twostep processing approach. First, we applied a median filtering (21 samples; empirically defined) in order to remove high-frequency noise and smooth the trend of the features' values over time. This provided us with a robust way to deal with the noise in features' values due to noisy ECG signals. Second, we applied a moving window (31 samples; defined empirically) over the filtered signal and calculated the distribution parameters in every window, obtaining in that way a longitudinal trajectory. Figure 1 shows an example of this two-step approach for one of the features (STE III) and for one of the patients in the dataset.

\section{Results}

Figure 2 shows an example of the temporal evolution of the distribution parameters for all the features and for one of the patients in the STAFF III dataset. As can be observed, in the case of the standard deviation (upper left graph) and symmetry (bottom right graph) parameters, values at baseline are smaller and with less variation than in the AMI phase where the values significantly increase and decrease.

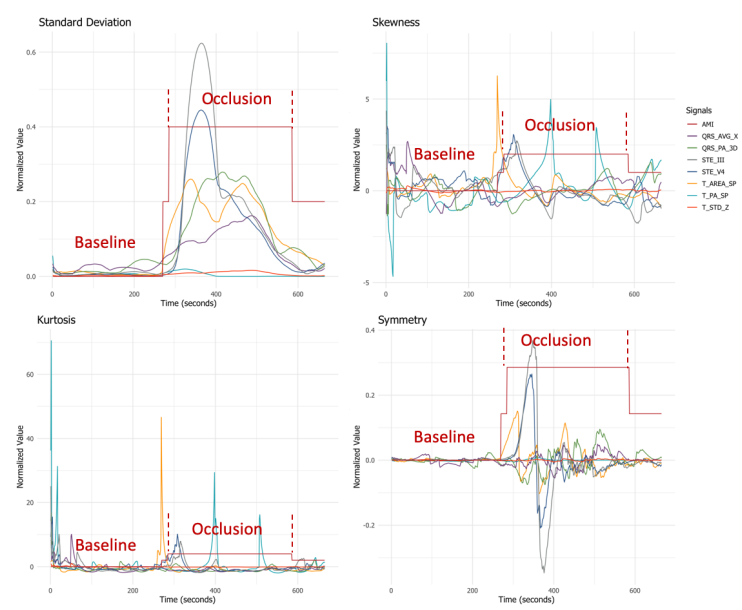

Figure 2 - Example of temporal evolution of distribution parameters for one of the patients in the STAFF III dataset. AMI signal indicates recording condition.

To study the differences in distribution parameters between baseline and AMI, we calculated the mean values of the different distribution parameters for the baseline and AMI situations. Figure 3 shows a box plot with those means for all the patients in the dataset, while Table 2 shows the p-values, calculated using a Wilcoxon signedrank test.

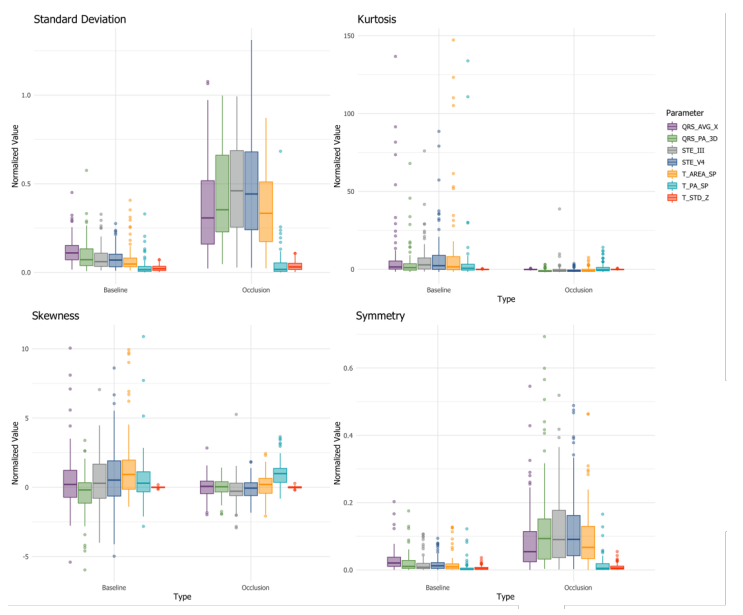

Figure 3 -Box plots of the mean values of distribution parameters during baseline and AMI (Occlusion). 
As can be observed in Figure 3, for the case of standard deviation and symmetry, there is a clear separation in mean values between baseline and AMI, what is confirmed by smaller p-values for most of the features in Table 2.

Table 2. Figure 3 boxplots p-values (Baseline vs AMI).

\begin{tabular}{lcccc}
\hline \multicolumn{1}{c}{ Feature } & $\begin{array}{c}\text { STD } \\
\text { p-value }\end{array}$ & $\begin{array}{c}\text { Skewness } \\
\text { p-value }\end{array}$ & $\begin{array}{c}\text { Kurtosis } \\
\text { p-value }\end{array}$ & $\begin{array}{c}\text { Symmetry } \\
\text { p-value }\end{array}$ \\
\hline T_PA_SP & 0.104 & 0.0413 & 0.0382 & 0.0191 \\
T_AREA_SP & $<0.0001$ & $<0.0001$ & 0.0002 & $<0.0001$ \\
T_STD_Z & 0.501 & 0.0921 & 0.1134 & 0.0827 \\
QRS_PA_3D & $<0.0001$ & 0.0127 & $<0.0001$ & $<0.0001$ \\
QRS_AVG_X & $<0.0001$ & 0.0234 & 0.0003 & $<0.0001$ \\
STE_III & $<0.0001$ & 0.0071 & $<0.0001$ & $<0.0001$ \\
STE_V4 & $<0.0001$ & 0.0018 & $<0.0001$ & $<0.0001$ \\
\hline
\end{tabular}

To further validate the performance of the distribution parameters, we used the standard deviation and symmetry parameters calculated over the significant features in Table 2 and built a model to differentiate between baseline and AMI situations. To reduce chances of overfitting, we divided the database into a training dataset with $70 \%$ of the patients (69 patients) and a test dataset with the other $30 \%$ ( 29 patients). We used the training dataset to build three models in order to discriminate between baseline and AMI situations. The first model uses the standard deviation parameter (with the ECG features mentioned before), the second model uses the symmetry parameter and the third model uses both, standard deviation and symmetry together. To build the models, and after trying different classification algorithms (KNN, lasso, gradient boosting and support vector machines), we decided to use gradient boosting method due to its good performance [9]. Once we trained the models, we used the test dataset for validation purposes.

Figure 4, shows the ROC curves with the performance of the three models in the validation dataset, while Table 3 shows the results from the significance analysis when comparing the performance of the three models with each other.

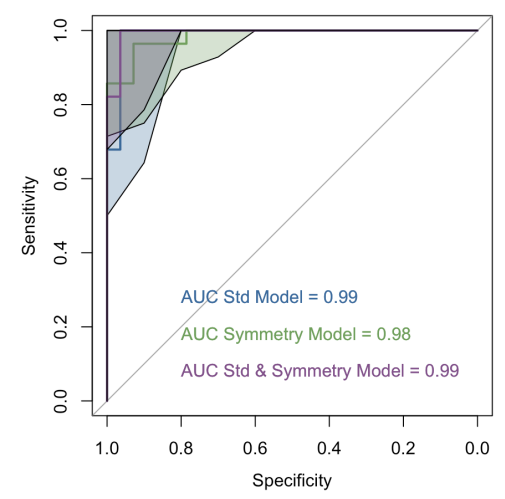

Figure 4 - Performance of models in the test dataset for the classification of baseline vs AMI.
Table 3. DeLong's test for AUC models.

\begin{tabular}{ll}
\hline Model & p-value \\
\hline Std vs Symmetry & 0.806 \\
Std vs (Std \& Symmetry) & 0.366 \\
Symmetry vs (Std \& Symmetry) & 0.465 \\
\hline
\end{tabular}

\section{Discussion}

In Figure 3 we observe differences in mean values of the distribution parameters (for the different features) between baseline and AMI conditions. Also, we observe that the best parameters to capture distribution differences between baseline and AMI are the standard deviation and symmetry. This is confirmed by the p-values in Table 2 . Additionally, we can see that not all the proposed features show significant differences ( $\mathrm{p}$-value $<0.05$ ), being T PA SP and T STD Z not significant features for the standard deviation and symmetry distribution parameters. It is worth noticing that in [6], T_PA_SP and T_STD_Z were selected as ones of the best features from a total of 328 features, while in this new research they are not. We believe the reason for that is that in [6], we solved a classification problem and compared features values in 10 seconds intervals at baseline and AMI, while in this work we analyzed changes over time in the distribution properties of the features during baseline and AMI. Therefore, since we are looking to different problems, this could explain the differences in the results we obtained in these two studies.

Also, for standard deviation and symmetry parameters, the distributions at baseline are narrower than during AMI. A narrower standard deviation during baseline may be explained by a stable condition, where features' values show small variability. On the other hand, a more spread standard deviation during AMI could be explained by an unstable condition, where there are changes over time on the ECG (due to heart condition changes), that are reflected as an increase standard deviation in features' values. For the symmetry parameter a similar reasoning applies, smaller values at baseline indicates that there is not much change in distributions' shape (mean and median are similar), while bigger values during AMI, indicates changes in distributions' shape, most likely because of the shifting of features' values over time due to the AMI condition. In addition to that, in Figure 2 we can observe that when AMI condition starts, there is a transition phase in which values start to increase for the standard deviation and oscillate for the symmetry parameter. This behavior is nothing specific of the concrete patient example in Figure 2 , but something present in all the patients in the dataset. This shows the potential of those parameters in the dynamic assessment of AMI since they appear to be able to capture the dynamic nature underlying the AMI process. We believe that this characteristic will allow to reduce the false positive rates, since AMI condition could be detected by analyzing parameters values in reference to prior trends and trajectories for the specific patient, instead 
of considering independent measurements and comparing them with the average AMI patient.

Lastly, standard deviation and symmetry parameters, showed good performance for discriminating between baseline and AMI situations. The performance of the three models was similar (Figure 4), performing best the combination of both standard deviation and symmetry parameters (AUC 0.99), followed by the standard deviation (AUC 0.99) and symmetry (AUC 0.98). Nonetheless, the differences in performance were small and not significant (Table 3 ).

We believe that, all the findings exposed in this section, together with the fact that we used a set of features derived from a reduced three ECG lead system, makes the proposed method suitable for the continuous and dynamic monitoring of AMI in long-term, ambulatory and remote-monitoring conditions. Even more, this method analyzes changes over time and therefore it does not strictly need a prior patient baseline since it could be built real-time, what we believe makes it usable in a clinical setting where most of the times a prior baseline for the patient does not exist. This will help in reducing false positive rates since will enable to create a specific baseline for each patient. Also, the decision will be made based on the evolution of the patient's parameters trends instead of independent patient's values. Nonetheless, still more work must be done in order to explore the longitudinal capabilities of the distribution parameters for the dynamic assessment of AMI condition.

\section{Conclusions}

In this work we proposed a novel set of parameters which we hope will help in the dynamic assessment of AMI condition in patients. Those parameters were obtained from the analysis of changes over time in the distribution properties of a set of ECG features derived from a reduced 3-lead ECG system. The best parameters to differentiate between baseline and AMI situations were standard deviation and symmetry. These parameters showed a transition phase, starting with the beginning of the AMI condition, in which standard deviation values increase and symmetry values oscillate. This property of the parameters makes us think that they could be used in the dynamic assessment of AMI since they appear to be able to capture the dynamic nature underlying the AMI process. This opens the possibility to detect the AMI condition by comparing actual parameter values with prior trends of the patient (patient base approach), which will help in reducing the false positive rates.

Also, we evaluated the capabilities of the method to discriminate between baseline and AMI situations using for that three different models. The good results obtained in all the models, suggests that indeed, distribution parameters are able to accurately differentiate between baseline and AMI situations. This finding, together with the capabilities of the distribution parameters to capture the dynamic behavior of the AMI condition, and the robustness of those parameters against ECG noise, make those parameters good candidates to reduce false alarms rate and maybe to be implemented in the clinical practice. Even more, given that we used a reduced lead system, this method is also suitable for continuous and dynamic monitoring of AMI in long-term, ambulatory and remotemonitoring conditions, making possible an earlier diagnosis of AMI.

One of the limitations of this work is related with the used dataset, which contains simulated AMI events due to coronary artery occlusion during a PTCA procedure. Therefore, it could be that this dataset does not reflect a real AMI process and the ECG changes associated to it. Some more analysis has to be done in order to validate the AMI model in the STAFF III dataset with a real AMI dataset.

Finally, more work needs to be done in order to evaluate the false alarm rate and to explore the longitudinal capabilities of the distribution parameters in the dynamic assessment of AMI.

\section{References}

[1] Thygesen, K., Alpert, J. S., Jaffe, A. S., Simoons, M. L., Chaitman, B. R., White, H. D., Wagner, D. R. (2012). Third universal definition of myocardial infarction. Circulation, 126(16), 2020-2035.

[2] Ansari, S., Farzaneh, N., Duda, M., Horan, K., Andersson, H. B., Goldberger, Z. D. and N. K. (2017). A review of automated methods for detection of myocardial ischemia and infarction using electrocardiogram and electronic health records, IEEE Review.

[3] Frank, E. (1956). An accurate, clinically practical system for spatial vectorcardiography. Circulation 13(5):e737-e749.

[4] Boutkan, J. (1965). Vectorcardiography: physical bases and clinical practice. First ed. Eindhoven: Centrex Publishing Company, 1965.

[5] Aranda, A., Bonizzi, P., Karel, J., Peeters, R. (2015). Performance of Dower's Inverse Transform and Frank Lead System for Identification of Myocardial Infarction. Engineering in Medicine and Biology Society (EMBC), 2015 37th Ann. Int. Conf. of the IEEE. pp. 4495-4498.

[6] Aranda Hernandez, A., Bonizzi, P., Karel, J., Peeters, R. (2018). Myocardial Ischemia Diagnosis Using a Reduced Lead System. Engineering in Medicine and Biology Society (EMBC), 2018 40th Ann. Int. Conf. of the IEEE. pp. 5302-5305.

[7] Goldberger, A., Amaral, L., Glass, L., Hausdorff, J., Ivanov, P., Mark, R., et al. (2000). PhysioBank, PhysioToolkit, and PhysioNet: components of a new research resource for complex physiologic signals. Circulation 101(23):e215-e220

[8] Martínez, J. P., Pahlm, O., Ringborn, M., Warren, S., Laguna, P., Sörnmo L. (2017). The STAFF III database: ECGs recorded during acutely induced myocardial ischemia. Comput Cardiol, 44:266-133.

[9] Greg Ridgeway et al. (2017). gbm: Generalized Boosted Regression Models. R package version 2.1.3. https://CRAN.Rproject.org/package $=\mathrm{gbm}$

Address for correspondence:

Alfonso Aranda

Endepolsdomein 5, Maastricht (6229GW), The Netherlands

a.arandahernandez@maastrichtuniversity.nl 